\title{
Research into Excited Long Lived 0.6-6.0 keV Energy Levels in the Cathode Solid Medium of Glow Discharge by X-Ray Spectra Emission
}

\author{
Alexander Borisovich Karabut \\ FSUE SIA LUTCH, Podolsk 140055, Moscow Region, Russia.
}

Received: January 31, 2013 / Accepted: February 27, 2013 / Published: May 25, 2013.

\begin{abstract}
X-ray emissions have been registered in experiments with high-current glow discharge from the different cathode and different plasma forming gases within $100 \mathrm{~ms}$ and more after turning off the current. The emission X-rays was realized: in the form of laser microbeams (up to $10^{4}$ beams a second and up to $10^{10} \mathrm{X}$-ray of quanta in a beam, angular divergence was up to $10^{-4}$ ). The fine structure of X-ray spectra were detected using a bent mica crystal X-ray spectrometer and recorded on X-ray film after the discharge current switching off (during up to $20 \mathrm{~h}$ afterwards). The X-ray energy was 0.8-6.0 keV. The possible mechanism and phenomenon model of producing the X-ray emission on the solid medium cathode with the exited energy levels was considered.
\end{abstract}

Key words: High-current glow discharge, solid medium cathode, X-ray emission.

\section{Introduction}

The experiments on defining a possible mechanism of high energy phenomena in the solid-state cathode medium of the high-current glow-discharge were carried out before. The generation of penetration radiation was registered by the researches of high energy process in solid cathodes of electric discharge [1]. The experiments were carried out on the high-current Glow Discharge device in $\mathrm{D}_{2}, \mathrm{H}_{2}, \mathrm{He}, \mathrm{Kr}$ and $\mathrm{Xe}$ at pressure up to 10 Torr, current up to 300 $\mathrm{mA}$ and the Glow Discharge voltage ranging 1,500-4,300 V. The cathode samples being made of Al, $\mathrm{Sc}, \mathrm{Ti}, \mathrm{Ni}, \mathrm{Nb}, \mathrm{Zr}, \mathrm{Mo}, \mathrm{Pd}$ and $\mathrm{Ta}, \mathrm{W}$. The registration of X-ray emission was performed using $\mathrm{Al}_{2} \mathrm{O}_{3}$-based TLD (Thermo-Luminescent Detectors), the pine hole and PM scintillating detectors for the recording of temporal characteristics [2-7]. The following modes were brought during the experiments: (1) diffusion

Corresponding author: Alexander Borisovich Karabut, doctor, Ph. D., research fields: plasma, laser and nuclear physics. E-mail: abkarab@mail.ru.
X-ray emission was observed in the form of separate $\mathrm{X}$-ray flashes (up to $10^{5} \mathrm{X}$-ray in flashes/s and up to $10^{4}$ X-ray photons in a flash), (2) X-ray beams emission by small angular divergence occurs during the discharge burning and up to $100 \mathrm{~ms}$ and more after the current turning out. The X-ray spectra were registered in film using the curved mica crystal X-ray spectrometer. The X-ray spectrum were registered both as bands of the continuum with energies ranging 0.6-10.0 keV and as spots resulting from the emission of series of high-density monoenergetic X-ray beams (with energies of $0.6-10.0 \mathrm{keV}$ ) characterized by small angular divergence. The spectra were repeatedly recorded during the glow discharge operation and after the glow discharge current switch off (for up to $20 \mathrm{~h}$ afterwards) [9]. The results obtained with different types of detectors are related to each other well. All the experimental results have 100\% reproducibility. Experiment on the $\mathrm{X}$ in the absence of current electrical discharge of particular importance for a variety of application uses. 


\section{Experiments}

\subsection{Glow Discharge Device}

The measurements were carried out using the glow discharge device consisting of a water-cooled vacuum chamber, the cathode and the anode assemblies (Fig. 1a). The cathode design allowed the placement of cathode samples made of various materials on a water-cooled surface. The experiments were carried out using a high-current glow discharge in $\mathrm{D}_{2}, \mathrm{H}_{2}, \mathrm{He}$, $\mathrm{Kr}$ and $\mathrm{Xe}$ and the cathode samples being made of $\mathrm{Al}$, $\mathrm{Sc}, \mathrm{Ti}, \mathrm{Ni}, \mathrm{Nb}, \mathrm{Zr}, \mathrm{Mo}, \mathrm{Pd}, \mathrm{Ta}$ and $\mathrm{W}$.

\subsection{Electric Discharge Power Supply}

A pulsed power supply was used in the experiments. The scheme of the pulse, periodical power supply was contained a sequence of high-voltage transistor switches, which are controlled from the driving pulse generator. Power supply contained five high-voltage transistor switches with operating voltages up to 4,500 $\mathrm{V}$ and current up to $300 \mathrm{~mA}$ (Fig. 1c).

\section{X-Ray Registration}

\subsection{X-Ray Scintillator-Photomultiplier Detectors}

The energy, spatial and time characteristics of the penetrating radiation were determined with use of the scintillation detectors supplied with the photomultipliers (PM). The signal from $\mathrm{PM}$ was transferred to a fast preamplifier and then to the two-channel computer digital oscillograph with the limit resolution frequency of $50 \mathrm{MHz}$ per a channel [5].

$\mathrm{X}$-ray emission in the form of laser beams was registered after passing the trailing edge of the glow discharge current pulses and turning glow discharge current. X-ray emission in the form of laser beams was registered for different cathode materials (Figs. 2a, 2b) and different gazes (Fig. 3).

\subsection{X-Ray Energy Measurements.}

The intensity of the luminous flux from the scintillator in the mode of generating the X-ray laser beams was very much. In this case, the amplification constant of the radiation recording system changed by changing the supply voltage of the photomultiplier and changing the amplification constant of the oscillograph [8]. Under some experiments the luminous-absorbing filter attenuating the luminous flux coming to PM was installed between the scintillator and PM. Two types of the filters attenuating the luminous flux by 50 times and by 2500 times respectively were used. For the PM scintillator detector the relative intensity of the X-rays was determined as the total of the amplitudes $\Sigma_{\mathrm{Ai}}$ of all the X-ray bursts within the time interval of 1 second. The

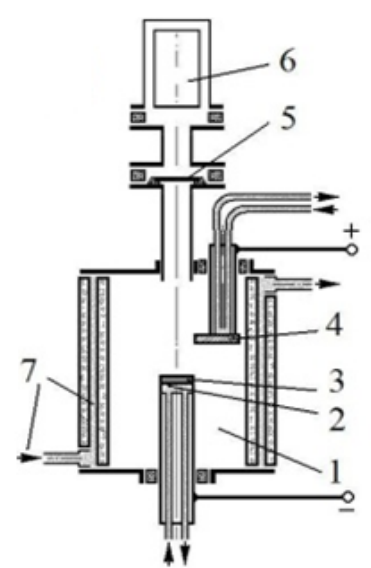

(a)

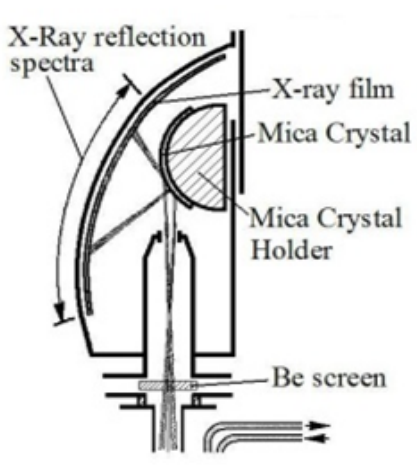

(b)

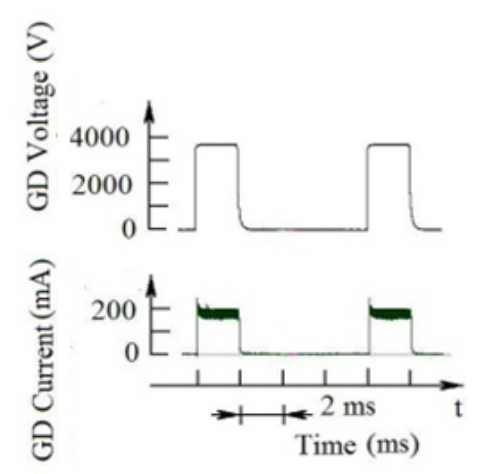

(c)

Fig. 1 Schematic representation of the experiment: (a) glow discharge device, (1) discharge chamber, (2) cathode holder, (3) cathode sample, (4) anode, (5) be foil screens, (6) X-ray scintillator, photomultiplier detectors, (7) cooling water; (b) X-ray spectrometer; (c) Glow discharge voltage and current oscillograms. 

Discharge by X-Ray Spectra Emission
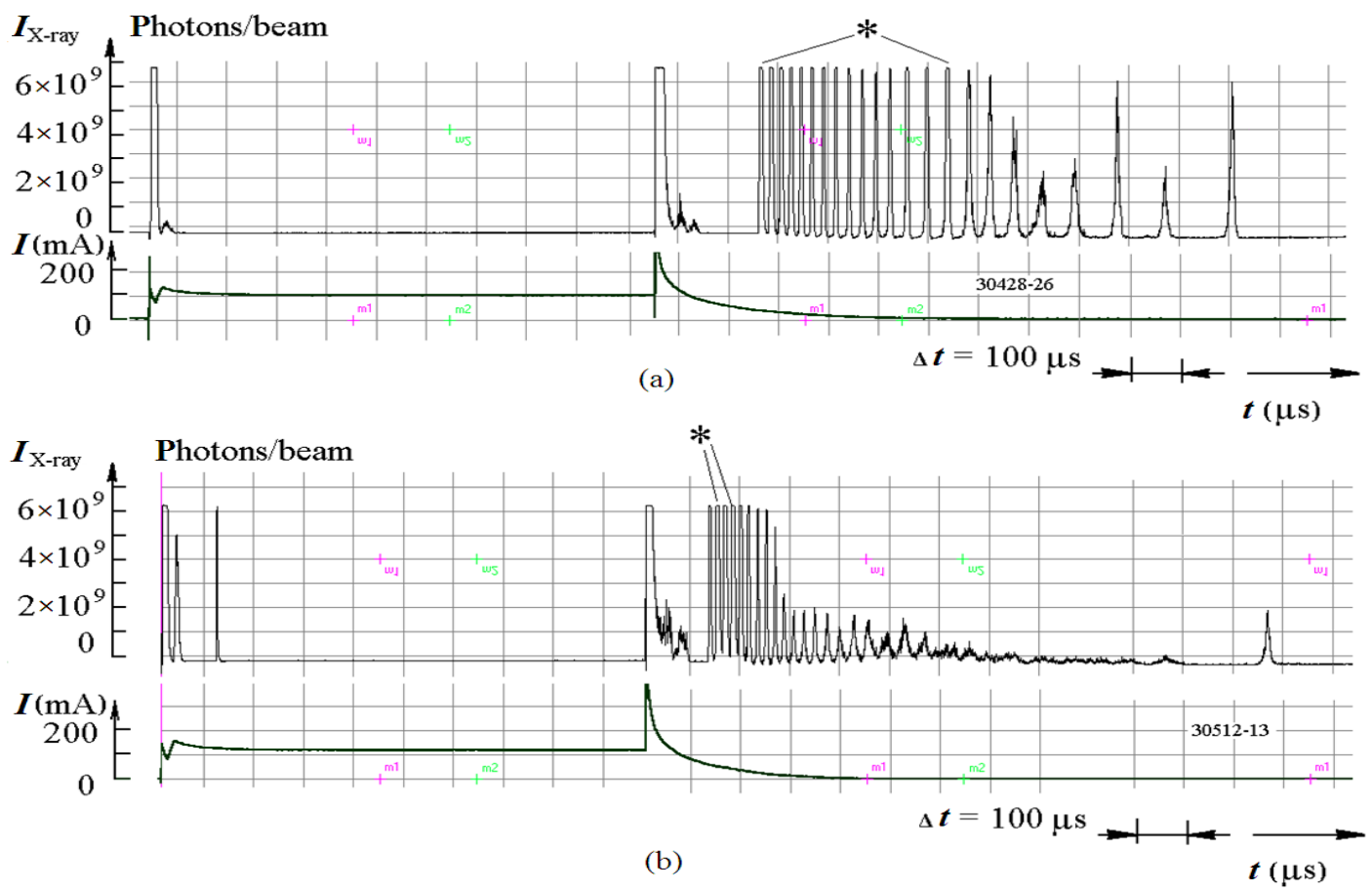

Fig. 2 The typical oscillograms of bursts from X-ray laser beams (PM, scintillator with optical filter) in the discharge for different kind of assemblyes. (a) the cathode sample is Ta; (b) the cathode sample is Mo, current, 100 mA, D2. (a) PM, scintillator arrange at a distance of $21 \mathrm{~cm}$ from cathode; (b) PM, scintillator arrange at a distance of $70 \mathrm{~cm}$ from cathode.

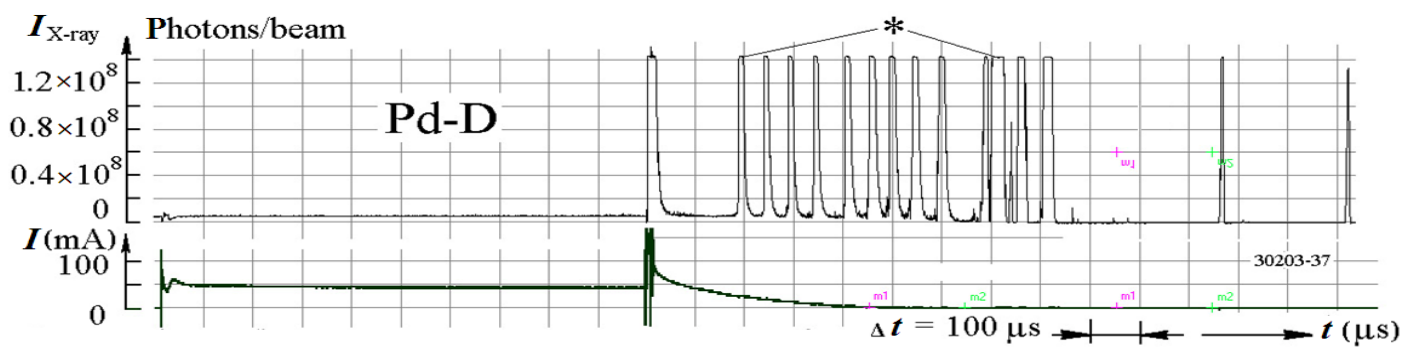

(a)

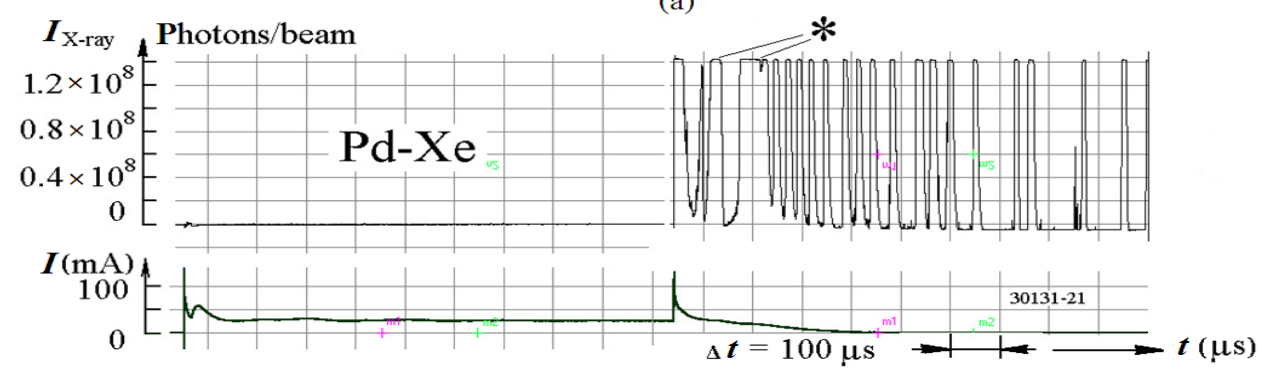

(b)

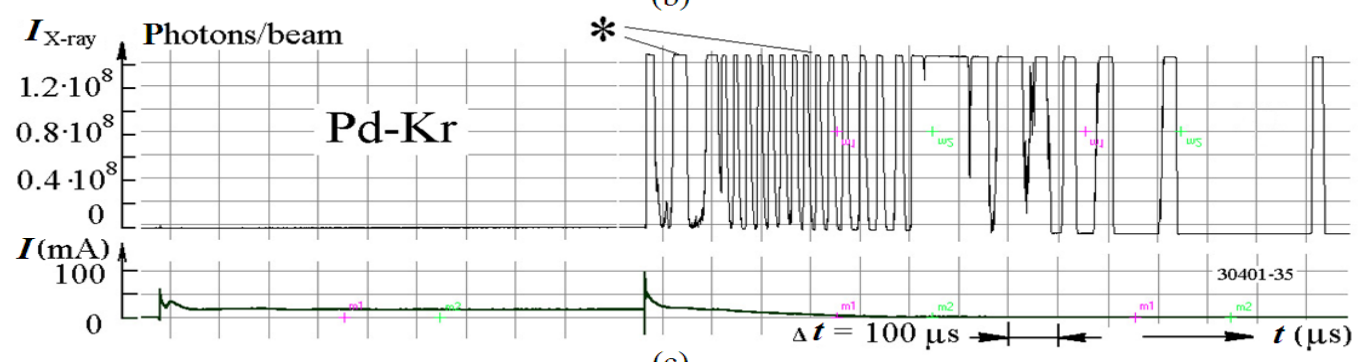

(c)

Fig. 3. The typical oscillograms of bursts from X-ray laser beams (PM, scintillator) in the discharge for different kind of gases. The cathode sample is Pd, current, $50 \mathrm{~mA}$. *, the pulse peak was cut a discriminator of amplifier. (a) D2, (b) Xe, (c) Kr. 
experiments with using the system of the scintillator, PM and shields made of the beryllium foil with thickness of $15 \mu \mathrm{m}$ and $30 \mu \mathrm{m}$ gave the assessment of the X-rays energy value of $E_{\mathrm{X} \text {-ray }} \approx 1.0-2.5 \mathrm{keV}$ (for different cathode materials) (Table 1).

\subsection{The X-Ray Emission Temporal Characteristics}

$\mathrm{X}$-ray emission as a function of time was studied with photomultiplier scintillator detectors.

The X-ray emission production begin after glow discharge the switching on in some time.

The time delay of X-ray emission production decrease for the increase of glow discharge current and voltage. The time delay of X-ray emission beginning is very small for high voltage glow discharge. Presumably the solid medium activation and the inverse population creation in solid after glow discharge switch on realize after some time.

The X-ray emission production occurred during the glow discharge operation, and, after the glow discharge current switch off. The generation of X-ray emission in the form of laser beams began when the glow discharge operational parameters increased (duration of current pulses, current density, GD voltage) and was observed as powerful flashes for the different cathode materials and for the different plasma forming gases. X-ray beams emission was registered through several time after passing the trailing edge of the Glow Discharge current pulses (Fig. 4). The different values of the time delay was registered in experiments [9]. The temporal characteristic of X-ray beams emission was made for the following conditions: time zero point correspond of the trailing edge time of the discharge current pulse, the number of X-ray beams was counted for each value of the time delay. The temporal characteristic of $\mathrm{X}$-ray beams emission show what the time delay are the fix values with the $\pm 2 \mu$ s deviation (Fig. 5).

\section{X-Ray Spectra Registration after Glow Discharge Switch off}

The X-ray emission spectra were measured using the curved mica crystal X-ray spectrometer (the mica crystal holder is $50 \mathrm{~mm}$ diameter), with the spectrum being registered on X-ray film [9, 10]. The film includes the refraction spectra, the direct X-ray lighting, and reflection spectra, with reflection spectra used for data processing. The direct X-ray lighting limits the reflection spectra in the high energy area. The wavelength and the energy of the X-ray were determined according to the expression: $m \lambda=2 \mathrm{~d} \sin \theta$;

$$
E_{\mathrm{X} \text {-ray }}=1.235 / \lambda
$$

Table 1 X-Ray photon energy for discharge current absence.

\begin{tabular}{lcccccccccc}
\hline Material of Cathode & $\mathrm{Al}$ & $\mathrm{Sc}$ & $\mathrm{Ti}$ & $\mathrm{Ni}$ & $\mathrm{Mo}$ & $\mathrm{Pd}$ & $\mathrm{Ta}$ & $\mathrm{Re}$ & $\mathrm{Pt}$ & $\mathrm{Pb}$ \\
\hline Glow discharge voltage $(\mathrm{V})$ & 1,650 & 1,540 & 1,730 & 1,650 & 1,420 & 1,650 & 1,600 & 1,520 & 1,650 & 1,610 \\
$\begin{array}{l}\text { Glow discharge current }(\mathrm{mA}) \\
130\end{array}$ & 130 & 170 & 150 & 210 & 138 & 38 & 125 & 138 & 138 \\
$\begin{array}{l}\text { X-ray energy, current switch } \\
\text { off, } E_{\text {X-ray }}(\mathrm{keV})\end{array}$ & 1.68 & 1.5 & 1.46 & 1.96 & 1.33 & 1.71 & 1.62 & 1.38 & 1.75 & 1.45 \\
\hline
\end{tabular}

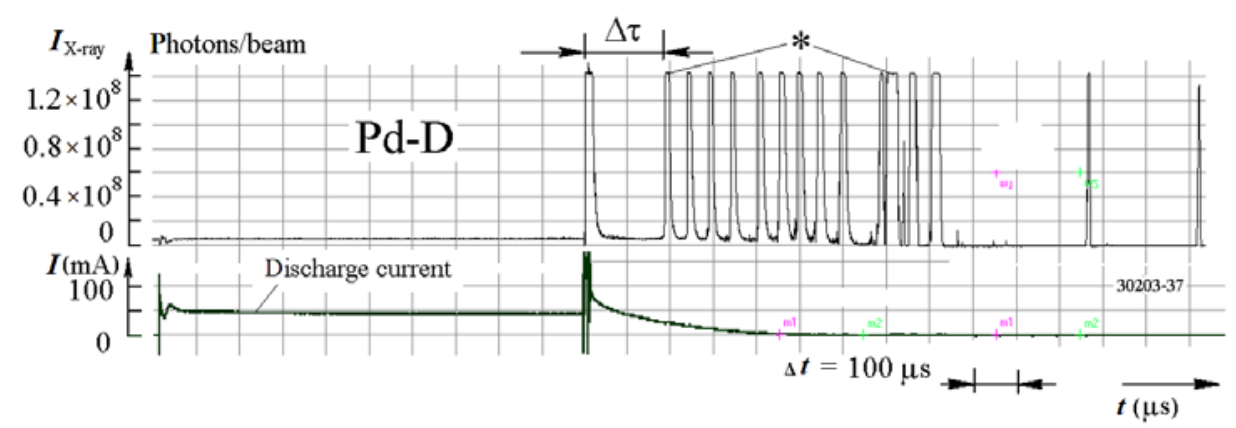

Fig. 4 The typical oscillograms of bursts from X-ray laser beams (PM, scintillator) after glow discharge turning. The cathode sample is Pd, D2 discharge current, $50 \mathrm{~mA}$. 


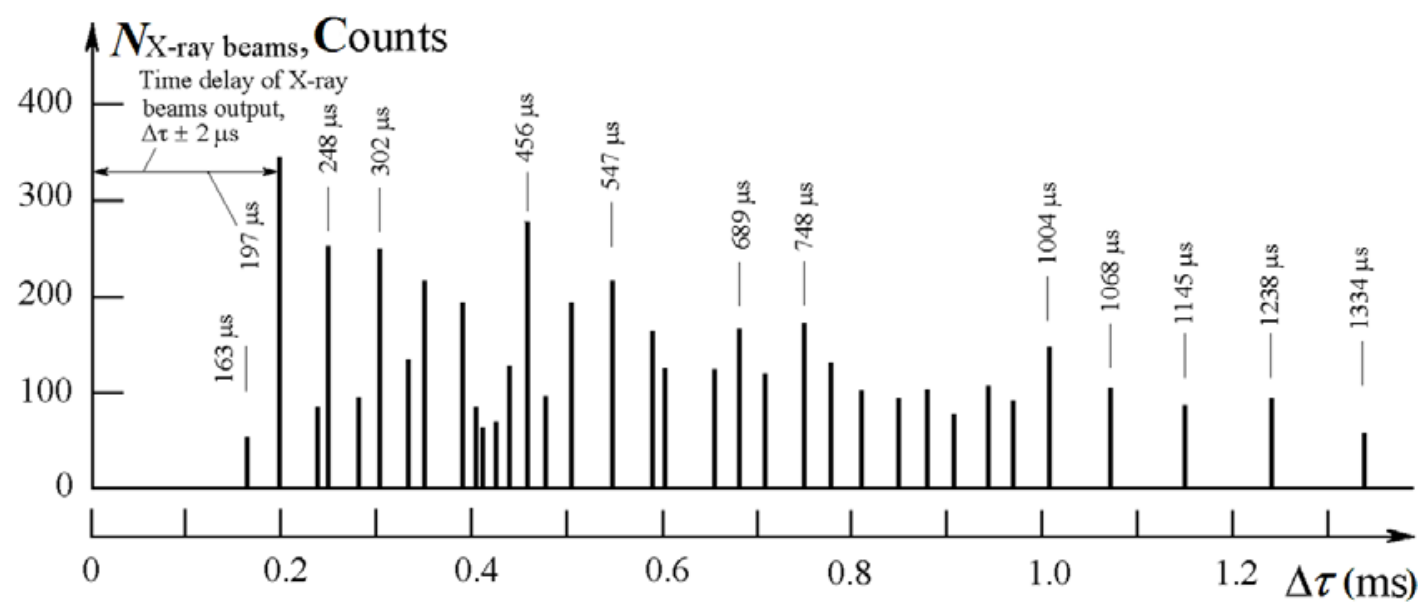

Fig. 5 Temporal characteristic of X-ray beams emission. The cathode sample is Pd, D2 discharge, current, $50 \mathrm{~mA}$.

where $m$ is the spectrum order; $\lambda$ stands for the X-ray emission wavelength in $\mathrm{nm} ; 2 d$ is the constant of the mica crystal lattice $(2 d=2.0 \mathrm{~nm})$; and $\theta$ represents the reflection angle. The spectra were repeatedly recorded during the glow discharge operation and after the glow discharge current switch off (for up to $20 \mathrm{~h}$ afterwards). The spectra spots were located in spectral areas specific for a given cathode material used (Figs. 6-9).

\section{Phenomenological Model of High Energy Cathode Processes}

$\mathrm{X}$-ray emission was registered from cathode electric discharge for electric current absents. These results are the consequence of a fundamental phenomenon unknown before: "Formation of a crystal lattice of a solid body when bombarding its surface by plasma ions of an electrical discharge of metastable excited long-living (up to tens ms and more) states with the excitation energy of 1-3 keV and more in a solid body". This phenomenon was registered in experiments for all cathode materials and all plasma forming gases [10]. Now opinion it exist that the electrons in metals carry relaxation of excited levels. Experiments with registration X-ray emission from the cathode electric discharge in the absence of discharge current allows to conclude that in metals can create the inverted population environment with energy 1-3 keV and more long-lived excited states.
Formation of excited energetic levels with such energy values are supposed to occur in processes of non-elastic interaction of the plasma ions of the electric discharge with electronic shells of the solid

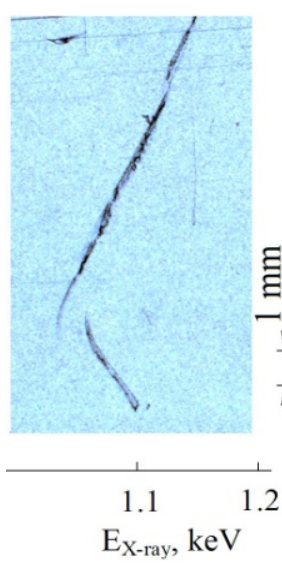

$\mathrm{Al}-\mathrm{He}$

Fig. $6 \mathrm{X}$-ray energy spectra in the spots and bands modes for Al cathode after the He glow discharge switch off. Exposition time is $20 \mathrm{~h}$.
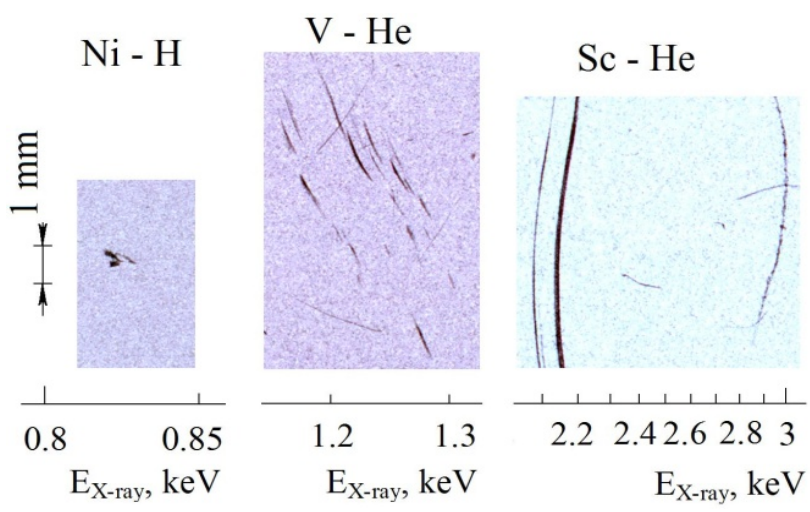

Fig. $7 \quad X$-ray energy spectra in the spots and bands modes for $\mathrm{Ni}, \mathrm{V}$ and Sc cathodes after the He glow discharge switch off. Exposition time is $20 \mathrm{~h}$. 


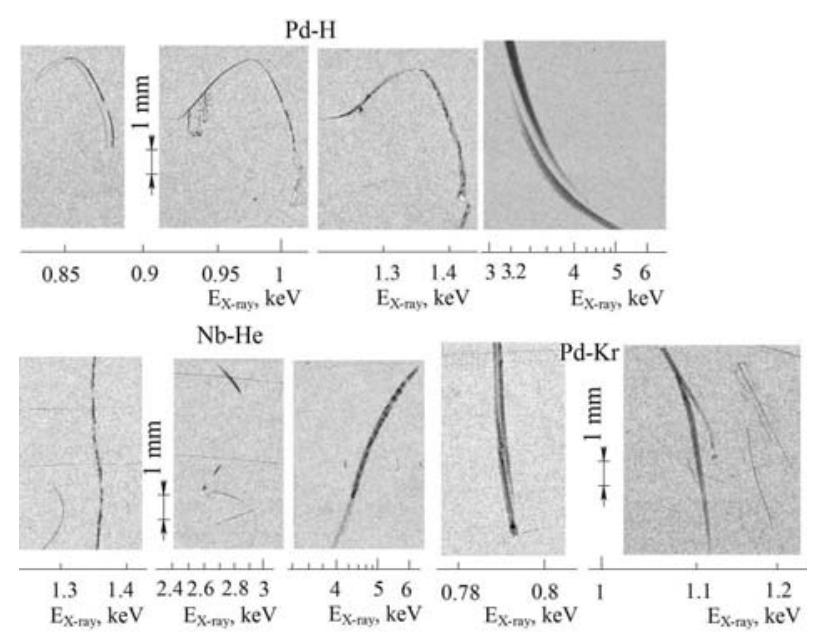

Fig. 8 X-ray energy spectra in the spot modes for different cathode materials after the glow discharge switch off. The cathode material and plasma gas forming was indicated. Exposition time is $20 \mathrm{~h}$.

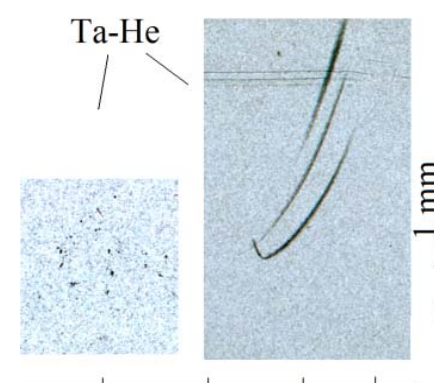

1.2

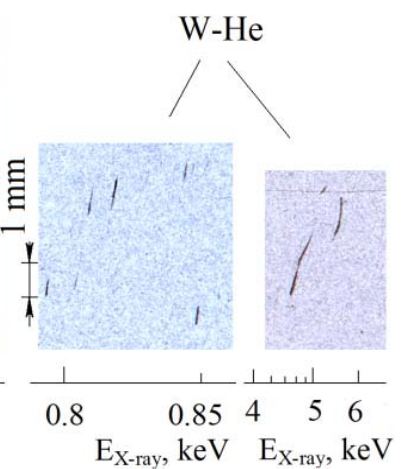

Fig. 9 X-ray energy spectra in the spots and bands modes for Ta and $\mathrm{W}$ cathodes after the He glow discharge switch off. Exposition time is $20 \mathrm{~h}$.

body ions. Fast relaxation of the metastable long-living excited ions states with the generation of $\mathrm{X}$-ray emission. The totality experimental results permit to consider the assume low energy nuclear reactions phenomenon theory in the solid medium of the electric discharge systems cathodes.

The main experimental result is following: The lifetime of the long-lived excited states with 1-3 keV energy in solid is possibly.

One can single out the following electric discharge systems areas and physical phenomena observed within them:

The obtained experimental results allow to consider the possible energy processes in the cathode material solid. The following processes may lead to initiation of low energy nuclear reactions:

- Deuterium ions acceleration in the Glow Discharge near-cathode area to energies from several hundred $\mathrm{eV}$ up to a few thousand $\mathrm{eV}$.

- Non-resilient processes of deuterium ions collision with the crystal lattice ions $\left(\mathrm{Pd}^{4+}\right.$ ions for Palladium). These processes cross-section related to non-adiabatic transition is determined according to massey criterion $\xi$. For $\Delta E=0.7-1.5 \mathrm{keV}$ (the near-cathode discharge conditions) the massey criterion $\approx 1$ and the non-resilient processes cross-section for optic polar phonons formation becomes close to gaso-kinetic one. Under these conditions the electron frame is displaced relative to nucleus and a dipole or optic polar phonon is formed. The initial optic polar phonons frequency is $\omega o$. At energies $W=1 \mathrm{keV} \omega o p=0.95 \times 1,019 \mathrm{~s}^{-1}$ which is higher than the plasma frequency $\omega p=(1.3-2.5) \times$ $1,016 \mathrm{~s}$, plasmones are not produced and there is no compensation for the arising spatial division of charges.

- At big concentration of initial optic phonons (high charge current density) non-linear unharmonic processes start playing their essential part. Presumably, processes of the 3 rd and the 4th order begin.

$$
\begin{gathered}
h \omega_{1 \text { in }}+h \omega_{2 \text { in }} \rightarrow h \omega_{3} \text { and } \omega_{1}<\omega_{3}>\omega_{2} \\
h \omega_{1 \text { in }}+h \omega_{2 \text { in }}+h \omega_{3 \text { in }} \rightarrow h \omega_{4} \text { and } \omega_{1}<\omega_{4}>\omega_{2}, \\
\omega_{4}>\omega_{3}
\end{gathered}
$$

These expressions mean that two $\left(h \omega_{1}\right.$ in, h $\omega_{2}$ in $)$ or three primary phonons are combined and formed into one $h \omega_{3}$ or $h \omega_{4}$. In this way out of a bog number of primary phonons with energies of $0.7-3.0 \mathrm{keV}$ a small number of high energy phonons (up to tens and hundreds $\mathrm{keV}$ ) is formed. The primary phonons energy spectrum is displaced in the direction of bigger values.

Presumably, some long-lived excited levels with energies up to several $\mathrm{keV}$ are formed in the cathode solid by the primary and secondary optic phonons energy. The excited energy levels for different materials are $0.8-6.0 \mathrm{keV}$. 
The non-equillibrium excited energy states with the population density of $\mathrm{n}$ exit $\left[\mathrm{cm}^{-3}\right]$ and a characteristic temperature of Texit $\approx 0.8-6.0 \mathrm{keV}$ were deexcited with X-ray emission.

\section{Conclusions}

The experimental research of this fundamental phenomenon has allowed to create an essentially new type of the device: "The X-ray solid-state laser with a wave length of the radiation of $0.2-1.5 \mathrm{~nm}$, duration of separate pulses of $10^{-11}-10^{-13} \mathrm{~s}$ and beam power in pulses up to $10^{7} \mathrm{~W}$ ". The obtained results show that creating optically active medium with long-living met stable levels with the energy of $1-3 \mathrm{keV}$ and more is possible in the solid.

\section{Acknowledgments}

Authors are happy to express their thanks to: professor Runar Kuzmin, solid and nuclear physic, Moscow State University, Sergey Pikuz, Doctor Ph.D., plasma physic, RAS Lebedev Physics Institute, George H. Miley, professor, plasma and nuclear physic, Ill. University, Peter L. Hagelstein, professor, plasma and laser physic, MIT for their help in this work.

\section{References}

[1] A.B. Karabut, Ya.R. Kucherov, I.B. Savvatimova, Nuclear reaction on in the cathode gas discharge, Pisma in GTF (Sov. Tech. Phys. Lett.) 16 (12) (1990) 53-57.

[2] A.B. Karabut, Ya.R. Kucherov, I.B. Savvatimova,
Nuclear product ratio for glow discharge in deuterium, Physics Letters A 170 (1992) 265-272.

[3] Ya.R. Kucherov, A.B. Karabut, I.B. Savvatimova, Metod and apparatus for long-term continuous energy production, US Patent, 93/08007 (1993).

[4] A.B. Karabut, Research into powerful solid X-ray laser (wave length is $0.8-1.2 \mathrm{~nm}$ ) with excitation of high current glow discharge ions, in: Proceedings of the 11 International Conference on Emerging Nuclear Energy Systems, Albuquerque, New Mexico, USA, 2002, pp. 374-381.

[5] A.B. Karabut, Experimental research into characteristics of X-ray emission from solid-state cathode medium of high-current glow discharge, in: Proceedings of the 10th International Conference on Cold Fusion, Cambridge, MA, USA, 2003, pp. 585-596.

[6] A.G. Lipson, A.S. Roussetski, A.B. Karabut, G.H. Miley, Gain of dd, reaction and X-ray emission generation in high current pulsed glow discharge in deuterium with $\mathrm{Ti}$, cathode at $0.8-2.45 \mathrm{kV}$ voltage, Journal of Experimental and Theoretical Physics, Russia 100 (2005) 1175-1189.

[7] A.B. Karabut, Experimental results on excess power, impurity nuclides, and X-ray production in experiments with a high-voltage electric discharge system, Journal Condensed Matter Nucl. Sci. 6 (2011) 1-20.

[8] A.B. Karabut, Excess heat production in $\mathrm{Pd} / \mathrm{D}$ during periodic pulse discharge current in various condition, in: Proceedings of the 11th International Conference on Cold Fusion, France, 2004, pp. 178-193.

[9] A.B. Karabut, Study of deuterium loading into pd cathode samples of glow discharge, in: Proceedings of 9th International Workshop on Anomalies in Hydrogen/Deuterium Gas Loaded Metals, Siena, Italy, 2010.

[10] A.B. Karabut, Research into excited 0.6-6.0 keV energy levels in the cathode solid medium of glow discharge by X-ray spectra emission, Journal Condensed Matter Nucl. Sci. 8 (2011) 1-17. 